\title{
A KEY METRIC AND ITS CALCULATION MODELS FOR A CONTINUOUS DIAGNOSIS CAPABILITY BASE DEPENDENCY MATRIX
}

\section{Jun-You Shi, Xie-Gui Lin, Meng Shi}

School of Reliability and System Engineering, Beihang University, Xueyuan Road 37, 100191 Beijing, China(ه jy.shi@yahoo.com, +8613661097725,linxg@dse.buaa.edu.cn,meng.shi016@gmail.com)

\begin{abstract}
This paper is devoted to measuring the continuous diagnosis capability of a system. A key metric and its calculation models are proposed enabling us to measure the continuous diagnosis capability of a system directly without establishing and searching the sequential fault tree (SFT) of the system. At first a description of a D matrix is given and its metric is defined to determine the weakness of a continuous diagnosis. Then based on the definition of a sequential fault combination, a sequential fault tree (SFT) is defined with its establishment process summarized. A key SFT metric is established to measure the continuous diagnosis capability of a system. Two basic types of dependency graphical models (DGMs) and one combination type of DGM are selected for characteristics analysis and establishment of metric calculation models. Finally, both the SFT searching method and direct calculation method are applied to two designs of one type of an auxiliary navigation equipment, which shows the high efficiency of the direct calculation method.
\end{abstract}

Keywords: fault diagnosis, dependency matrix, measurement.

(C) 2012 Polish Academy of Sciences. All rights reserved

\section{Introduction}

Dependency theory is a popular approach to testability design and analysis, and it has been given much more attention with respect to aviation equipment development in recent years [1-4]. In this theory, a dependency matrix (D matrix) is used to detect and isolate faults quickly. Thus, dependency theory is an effective way to implement continuous integrated diagnoses in prognostics and health management (PHM) systems.

A single-fault occurrence is a general assumption of dependency modeling [5-6]. Compared with the single-fault assumption, a multi-fault assumption reflects a practical situation better. Specifically, when a redundant system has fault-tolerant performance or when a complex system has no chance to be repaired during the operational process, multiple fault diagnosis (MFD) is worth considerable research effort [7, 8].

In the early 1990's, J.D. Kleer and B.C. Williams presented a method for MFD based on function models [9]. W.R. Simpson and J.W. Sheppard provided an algorithm for multiple fault isolation based on information flow models [10]. In the late 1990's and early 2000's, many optimal and near-optimal algorithms for MFD were presented, in which Lagrangian relaxation and subgradient optimization were used $[11,12]$. L. Tung and C.N. Hadjicostis presented max-product algorithms for the generalized multiple fault diagnosis (GMFD) problem [13]. M. Tadeusiewicz and S. Hałgas provided a method that enabled efficient identification of faulty elements and addressed MFD in analog AC or DC circuits [14]. Z. Wang and M. Marek-Sadowska changed the test sets used in the test and diagnosis to apply a simple single fault for MFD [15]. In recent years, new techniques for MFD were researched to improve diagnosis resolution [16]. D. Grzechca and J. Rutkowski applied the SVM 
(Support Vector Machine) algorithm to diagnosis and tests of analog electronic circuits with a high detection and localization level of circuit states [17]. With case-based reasoning and sequential fuzzy inference, several MFD methods were proposed [18, 19]. Using a selforganizing neural network, a methodology for parametric fault clustering in analog electronic circuits was proposed for fast and efficient circuit diagnosis [20]. Based on the principle of single-fault activation and single-output observation and a Boolean satisfiability solver, Y.C. Lin and F. Lu provided two fault diagnosis methods for improving MFD resolution [21]. M. Tadeusiewicz and S. Hałgas proposed a two-stage algorithm for multiple soft fault diagnosis of analog nonlinear circuits considering the component tolerances [22].

The above MFD methods focus on diagnosis at the end of a system operations process. When a continuous diagnosis is running during an operational process, multiple faults usually appear for which the faults occur one by one; these faults can be considered to be sequential faults [23]. In this case, the effects of previous faults play a major role, and the next diagnosis can be performed based on previous diagnosis results. A continuous diagnosis capability is an ability to continuously detect and isolate sequentially occurred faults without repairs in a system using a D matrix and D sub-matrixes. The process of a continuous diagnosis is as follows: first, a first occurred fault is diagnosed by a diagnosis strategy based on the D matrix; second, for the first occurred fault, a dependency sub-matrix (D sub-matrix) is established based on the available test-points left in the D matrix; after that, a second occurred fault can be diagnosed by a new diagnosis strategy based on the $\mathrm{D}$ sub-matrix; then the latter faults can be diagnosed continuously until a D sub-matrix cannot be established. Those diagnosed faults constitute a sequential fault combination in a diagnosed sequence. The length of the sequential fault combination reflects the continuous diagnosis capability of a system. By analyzing the processes of continuous diagnoses, all sequential fault combinations can be obtained. In the shortest sequential fault combinations, the units corresponding to the first occurred faults are considered to be the weaknesses of the continuous diagnoses.

In this paper, a key metric and its calculation models for a continuous diagnosis capability based on a D matrix are proposed. They enable us to measure the continuous diagnosis capability of a system directly without establishing and searching the sequential fault tree (SFT) of the system. At first a description of a D matrix and the definition of its metric are given in section 2. Then the definition of an SFT and its metrics are established in section 3. The characteristics of three types of dependency graphical models (DGMs) are analyzed with metric calculation models derived in section 4. The proposed metric and its calculation models are applied to two designs of one type of auxiliary navigation equipment in section 5 .

\section{D matrix and its metric}

\subsection{D matrix}

Dependency theory includes two models: a DGM and a D matrix [23]. A DGM of a system is a graphical description of the dependency relationships between the faults (which are marked as rectangles) and test-points (which are electrical connection points in the system for measurement, and marked as circles). A D matrix is a mathematical description of the above dependency relationships.

A D matrix is described by the following [24]:

$$
D_{m \times n}=F_{2} F_{1}\left[\begin{array}{cccc}
T_{1} & T_{2} & \cdots & T_{n} \\
\vdots & F_{m}
\end{array}\left[\begin{array}{cccc}
d_{11} & d_{12} & \cdots & d_{1 n} \\
d_{21} & d_{22} & \cdots & d_{2 n} \\
\vdots & \vdots & \ddots & \vdots \\
d_{m 1} & d_{m 2} & \cdots & d_{m n}
\end{array}\right],\right.
$$


where the $i$ th row $\left[d_{\mathrm{i} 1} d_{\mathrm{i} 2} \ldots d_{\mathrm{in}}\right]$ indicates the dependency relationships between the $i$ th fault $F_{\mathrm{i}}$ and all of the test-points $T_{\mathrm{j}}(j=1,2, \ldots \mathrm{n})$ and the $j$ th column $\left[d_{1 \mathrm{j}} d_{2 \mathrm{j}} \ldots d_{\mathrm{mj}}\right]^{T}$ indicates the dependency relationships between the $j$ th test-point $T_{\mathrm{j}}$ and all of the faults $F_{\mathrm{i}}(i=1,2, \ldots \mathrm{m})$. If $F_{\mathrm{i}}$ can be tested by $T_{\mathrm{j}}, d_{\mathrm{ij}}=1$; otherwise, $d_{\mathrm{ij}}=0$.

The D matrix can be obtained from the DGM and usually needs to be simplified to eliminate redundant information. The simplification process is given in literature [23] and can be summarized in the following steps:

1) Eliminate invalid test point;

2) Eliminate undetectable fault;

3) Combine ambiguity group.

\subsection{D matrix Metric}

Definition $1-\|F\|_{1}:\|F\|_{1}$ is defined as the 1-norm of a row vector in a $\mathrm{D}$ matrix. For the $i$ th row $\left[d_{\mathrm{i} 1} d_{\mathrm{i} 2} \ldots d_{\mathrm{in}}\right]$, its $\left\|F_{\mathrm{i}}\right\|_{1}$ is described as follows:

$$
\left\|F_{i}\right\|_{1}=\sum_{j=1}^{n}\left|d_{i j}\right| .
$$

$\left\|F_{\mathrm{i}}\right\|_{1}$ stands for the number of test-points that are dependent on $F_{\mathrm{i}}$. In the DGM of a system, the fault effect of $F_{\mathrm{i}}$ will flow through the model and can be detected by some test-points. The number of these test-points is equal to $\left\|F_{\mathrm{i}}\right\|_{1}$.

The weakness of a continuous diagnosis can be determined using $\left\|F_{\mathrm{i}}\right\|_{1}$. Given $F_{\mathrm{i}}$, if $\left\|F_{\mathrm{i}}\right\|_{1}$ is the maximum in all of the 1-norms of a $\mathrm{D}$ matrix, the module corresponding to $F_{\mathrm{i}}$ is a weakness.

\section{SFT and its metrics}

Based on the concepts given in literature [23], the more accurate description for sequential faults is presented as follows.

\subsection{SFT}

Definition 2 - Sequential fault combination: A sequential fault combination is defined as a set of faults (or fault ambiguity groups) that are diagnosed in sequence.

For each possible first diagnosed fault, a sequential fault combination can be achieved based on a D matrix.

Definition 3 - Sequential fault tree (SFT): An SFT is a tree description for all of the sequential fault combinations.

Fig. 1 shows an SFT example, in which each horizontal permutation represents a sequential fault combination, and there are totally four sequential fault combinations.

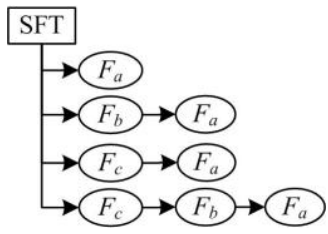

Fig. 1. The structure of a simple SFT.

To measure the continuous diagnosis capability of a system, all of the sequential fault combinations should be determined to establish the SFT. The main steps of establishing an 
SFT are as follows:

1) Establish the DGM of a system;

2) Establish the D matrix based on the DGM;

3) Determine all of the sequential fault combinations and establish an SFT.

\subsection{SFT Metrics}

Definition 4 - Branch: A sequential fault combination is defined as a branch in an SFT. For the SFT of a DGM with $k$ faults, let $b_{k}$ be the number of branches and $b_{k}$ equals the number of all of the sequential fault combinations.

Definition 5 - Node: A fault in a branch is defined as a node. For the SFT of a DGM with $k$ faults, let $N_{k}$ be the number of nodes in the SFT and $N_{k}$ equals the number of faults in all of the sequential fault combinations.

Definition 6 - $\boldsymbol{L}_{\text {avg }}: L_{\text {avg }}$ is the average length of branches in an SFT, which is described as follows:

$$
L_{\text {avg }}=\frac{N_{k}}{b_{k}} .
$$

In the above metrics, $L_{\mathrm{avg}}$ is the key metric.

\section{Characteristic analyses and metric calculation models of typical DGMs}

According to Section 3, we can establish and search the SFT to measure the continuous diagnosis capability of a system. This SFT searching method is suitable for all of the systems, but it has two disadvantages: 1) the efficiency is low; 2) it is very hard to establish an SFT for a large system. Therefore, metric calculation models should be established for measuring directly.

There are various DGM types for different systems, and most of them can be considered to be the combinations of series structures and parallel structures. Thus, two types of basic DGMs, series DGM and parallel DGM, are selected for fundamental research; and one type of combination DGM, fore-series-aft-parallel DGM, is selected for further research.

\subsection{Series DGM}

\subsubsection{Characteristic analysis of a series DGM}

In a series DGM, all of the faults are connected in series without feedback. Fig. 2 shows a series DGM with four faults connected in series. The D matrix and the SFT of this DGM are shown in Figs. 3 and 4.

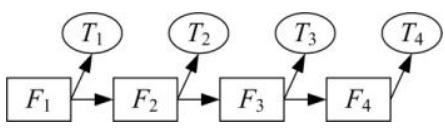

Fig. 2. A series DGM.

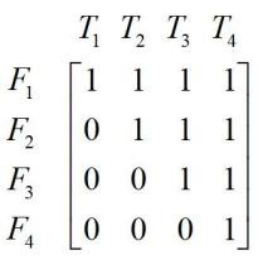

Fig. 3. D matrix of the series DGM.

As shown in Figs. 3 and 4, a series DGM has two important characteristics: 1) the D matrix is or can be converted into an upper triangular matrix based on the exchanging rule of rows or 
columns, and all of the elements above the main diagonal are equal to one; 2) in the SFT, when the arrangement of the first faults in all of the branches agrees with a decreasing order of $\|F\|_{1}$, the branches with a same first fault are generated by attaching all of the upper branches to this fault. The whole SFT can be generated by repeating this attaching process.

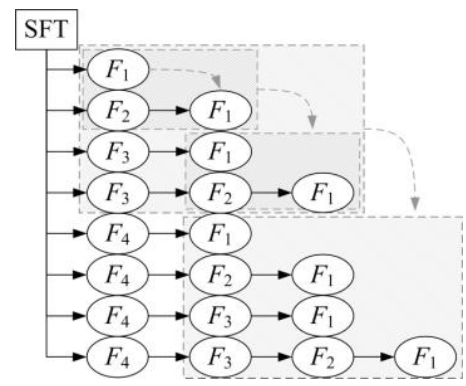

Fig. 4. SFT of the series DGM.

\subsubsection{Metric calculation models}

According to the above characteristics, the metric calculation models of a series DGM with $k$ faults can be derived as follows:

The D matrix of the series DGM is an upper triangular matrix, so the value of $\left\|F_{\mathrm{i}}\right\|_{1}$ decreases regularly with increasing $i$. In the case of Fig. 3, $k=4$ and $\left\|F_{1}\right\|_{1}=4,\left\|F_{2}\right\|_{1}=3,\left\|F_{3}\right\|_{1}=2$, $\left\|F_{4}\right\|_{1}=1$. Then the calculation model of $\left\|F_{\mathrm{i}}\right\|_{1}$ satisfies the following equation:

$$
\left\|F_{i}\right\|_{1}=k-i+1 \quad(i=1, \cdots, k)
$$

In the SFT of the series DGM, the branches with a same first fault are generated by attaching all of the upper branches to this fault. The relationship between $b_{k}$ and $k$ satisfies the following recurrence formula:

$$
\left\{\begin{array}{cc}
b_{k}=2 b_{k-1} & k>1 \\
b_{k}=1 & k=1
\end{array} .\right.
$$

Using mathematical induction, the calculation model of $b_{k}$ is derived from (5), which is described as follows:

$$
b_{k}=2^{k-1} .
$$

According to the above SFT characteristic, the relationship between $N_{k}$ and $k$ satisfies the following recurrence formula:

$$
\left\{\begin{array}{cc}
N_{k}=2 N_{k-1}+b_{k-1} & k>1 \\
N_{k}=1 & k=1
\end{array} .\right.
$$

Using mathematical induction, the calculation model of $N_{k}$ is derived from (7) and is described as follows:

$$
N_{k}=2^{k-2}(k+1) .
$$

Substituting (6) and (8) into (3) yields:

$$
L_{\text {avg }}=\frac{N_{k}}{b_{k}}=\frac{2^{k-2}(k+1)}{2^{k-1}}=\frac{k+1}{2} .
$$




\subsection{Parallel DGM}

\subsubsection{Characteristic analysis of a parallel DGM}

In a parallel DGM, all of the faults are connected in parallel. Fig. 5 shows a parallel DGM with four faults connected in parallel. The D matrix and the SFT of this DGM are shown in Figs. 6 and 7.

As shown in Figs. 6 and 7, a parallel DGM has two important characteristics: 1) the D matrix is or can be converted to a unit matrix, which is based on an exchanging rule for rows or columns; and 2) in the SFT, each branch is a full permutation result of all faults, and the whole SFT can be considered to be a set of all full permutation results.

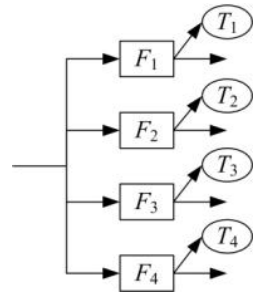

Fig. 5. A parallel DGM.

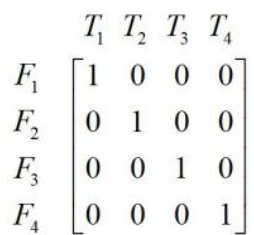

Fig. 6. D matrix of the parallel DGM.

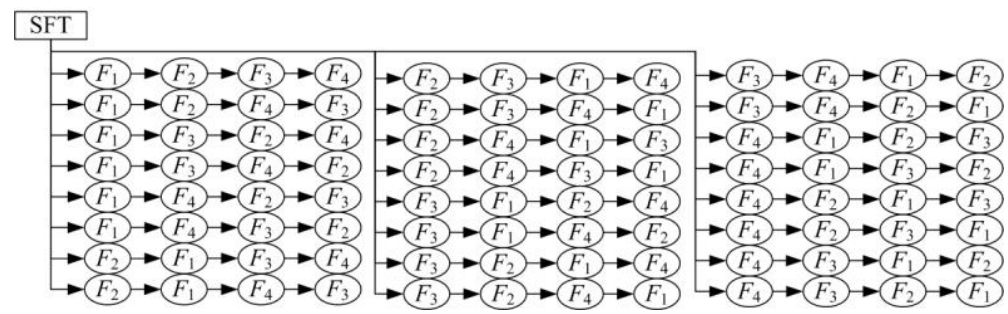

Fig. 7. SFT of the parallel DGM

\subsubsection{Metric calculation models}

According to the above characteristics, the metric calculation models of a parallel DGM with $k$ faults can be derived as follows:

The D matrix of the parallel DGM is a unit matrix, so the values of all $\left\|F_{\mathrm{i}}\right\|_{1}$ are the same. In the case of Fig. $6, k=4$ and $\left\|F_{1}\right\|_{1}=\left\|F_{2}\right\|_{1}=\left\|F_{3}\right\|_{1}=\left\|F_{4}\right\|_{1}=1$. Then the calculation model of $\left\|F_{\mathrm{i}}\right\|_{1}$ satisfies the following equation:

$$
\left\|F_{i}\right\|_{1}=1 \quad(i=1, \cdots, k) .
$$

In the SFT of the parallel DGM, each branch is a full permutation result of all faults. The relationship between $b_{k}$ and $k$ satisfies the following equation:

$$
b_{k}=P_{k}^{k}=k \text { ! }
$$

According to the full permutation characteristic, each branch has the same number of faults, i.e., $k$ faults. The number of nodes in the SFT is the product of $b_{k}$ and $k$, so the relationship between $N_{k}$ and $k$ satisfies the following equation: 


$$
N_{k}=b_{k} \times k=k \times k !
$$

Under this model, each branch is a full permutation result of all of the faults, so $L_{\text {avg }}$ satisfies the following equation:

$$
L_{\text {avg }}=k \text {. }
$$

\subsection{Fore-series-aft-parallel DGM}

\subsubsection{Characteristic analysis of a fore-series-aft-parallel DGM}

A fore-series-aft-parallel DGM can be divided into two sub-DGMs. One is a series DGM at the front of the whole DGM, the other is a parallel DGM at the back of the whole DGM.

Fig. 8 shows a fore-series-aft-parallel DGM in which two faults are connected in series with two faults connected in parallel. The D matrix and the SFT of this DGM are shown in Figs. 9 and 10.

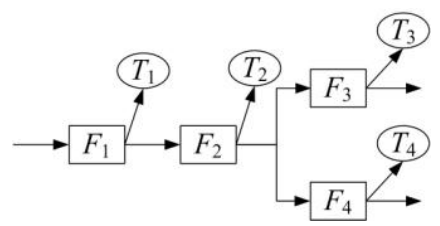

Fig. 8. A fore-series-aft-parallel DGM.

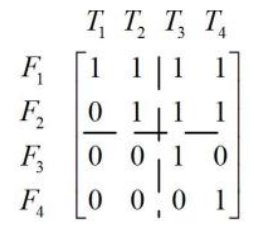

Fig. 9. D matrix of the fore-series-aft-parallel DGM.

As shown in Figs. 9 and 10, a fore-series-aft-parallel DGM has two important characteristics: 1) the $\mathrm{D}$ matrix is a combination of a 0-matrix, a 1-matrix and the $\mathrm{D}$ matrixes of two sub-DGMs. And it is or can be converted into an upper triangular matrix; and 2) the whole SFT can be considered to be an interlaced combination of two sub-SFTs. The first subSFT is the standard SFT of a series DGM, which is shown in Fig. 11(a). The second sub-SFT is a sum of the standard SFT of a parallel DGM and all SFTs of possible sub-parallel cases, which is shown in Fig. 11(b).

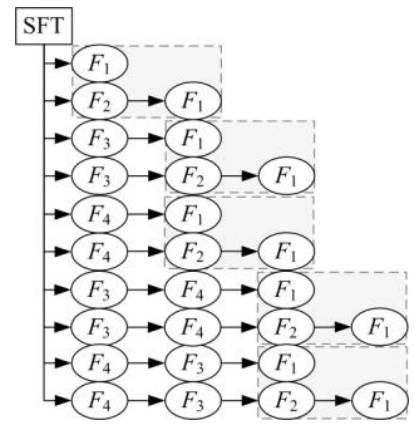

Fig. 10. SFT of the fore-series-aft-parallel DGM.

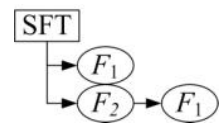

(a) The first sub-SFT

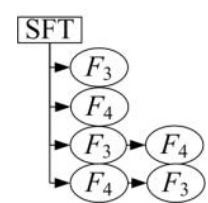

(b) The second sub-SFT.

Fig. 11. Two sub-SFTs 


\subsubsection{Metric calculation models}

According to the above characteristics, the metric calculation models of a fore-series-aftparallel DGM in which $k 1$ faults are connected in series with $k 2$ faults connected in parallel can be derived as follows:

Set $k=k 1+k 2$. According to the structure of the D matrix, $\left\|F_{\mathrm{i}}\right\|_{1}$ can be calculated by the following equation:

$$
\left\|F_{i}\right\|_{1}=\left\{\begin{array}{cc}
k-i+1 & (i=1, \cdots, k 1) \\
1 & (i=k 1+1, \cdots, k)
\end{array} .\right.
$$

Let $b_{k 1}$ be the number of branches in the first sub-SFT. The first sub-SFT is a standard SFT of the series DGM, so the relationship between $b_{k 1}$ and $k 1$ satisfies the following equation:

$$
b_{k 1}=2^{k 1-1} \text {. }
$$

Let $b_{k 2}$ be the number of branches in the second sub-SFT. The second sub-SFT is a sum of a standard SFT of the parallel DGM and all SFTs of possible sub-parallel cases, so the relationship between $b_{k 2}$ and $k 2$ satisfies the following equation:

$$
b_{k 2}=\sum_{i=1}^{k 2} P_{k 2}^{i} .
$$

The whole SFT is an interlaced combination of the two sub-SFTs, so the total number of branches $b_{k}$ satisfies the following calculation model:

$$
b_{k}=\left(1+b_{k 2}\right) \times b_{k 1} .
$$

Substituting (15) and (16) into (17) yields:

$$
b_{k}=\left(1+\sum_{i=1}^{k 2} P_{k 2}^{i}\right) \times 2^{k 1-1} .
$$

Let $N_{k 1}$ be the number of nodes in the first sub-SFT. According to (8), the relationship between $N_{k 1}$ and $k 1$ satisfies the following equation:

$$
N_{k 1}=2^{k 1-2} \times(k 1+1) \text {. }
$$

Let $N_{k 2}$ be the number of nodes in the second sub-SFT. Based on (12), the relationship between $b_{k 2}$ and $k 2$ is derived as follows:

$$
N_{k 2}=\sum_{i=1}^{k 2}\left(i \times P_{k 2}^{i}\right) .
$$

Based on the relationship of the interlaced combination, the total number of nodes $N_{k}$ satisfies the following calculation model:

$$
N_{k}=N_{k 1} \times\left(b_{k 2}+1\right)+b_{k 1} \times N_{k 2} .
$$

Substituting (15), (16), (19) and (20) into (21) yields:

$$
N_{k}=2^{k 1-1} \times\left[\sum_{i=1}^{k 2} i \times P_{k 2}^{i}+\frac{(1+k 1)}{2}\left(1+\sum_{i=1}^{k 2} P_{k 2}^{i}\right)\right] .
$$

Then a calculation model of $L_{\mathrm{avg}}$ is derived as follows: 


$$
L_{\text {avg }}=\frac{N_{k}}{b_{k}}=\frac{1+k 1}{2}+\frac{\sum_{i=1}^{k 2} i \times P_{k 2}^{i}}{1+\sum_{i=1}^{k 2} P_{k 2}^{i}} .
$$

\section{Case study}

One type of auxiliary navigation equipment is used to calculate navigation parameters for some aircraft. This navigation equipment must implement a continuous diagnosis during its operations process. The navigation equipment has two designs with six function modules, which are as follows: Anti-shake module, High voltage module, Stable frequency module, Basic output module, Satellite communication module and Standard output module. For convenience, the faults of the six modules are marked sequentially as $F_{1}, F_{2}, F_{3}, F_{4}, F_{5}$ and $F_{6}$, and the output port test-points of the faults are marked sequentially as $T_{1}, T_{2}, T_{3}, T_{4}, T_{5}$ and $T_{6}$ in the DGMs of both designs.

\subsection{Design-I}

The DGM and corresponding D matrix are shown in Figs. 12 and 13. The DGM of DesignI is a typical fore-series-aft-parallel DGM, in which three faults are connected in series with three faults connected in parallel, i.e., $k 1=k 2=3$.

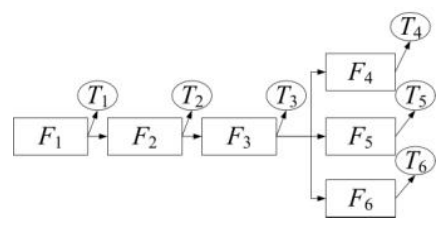

Fig. 12. The DGM of Design-I.

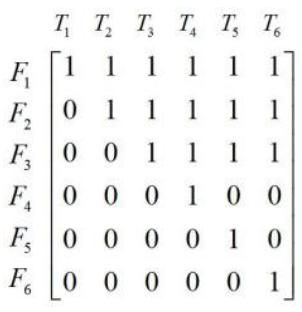

Fig. 13. The D matrix of Design-I.

\subsubsection{SFT searching method}

Using the SFT searching method, we have established the SFT of Design-I, which is shown in Fig. 14.

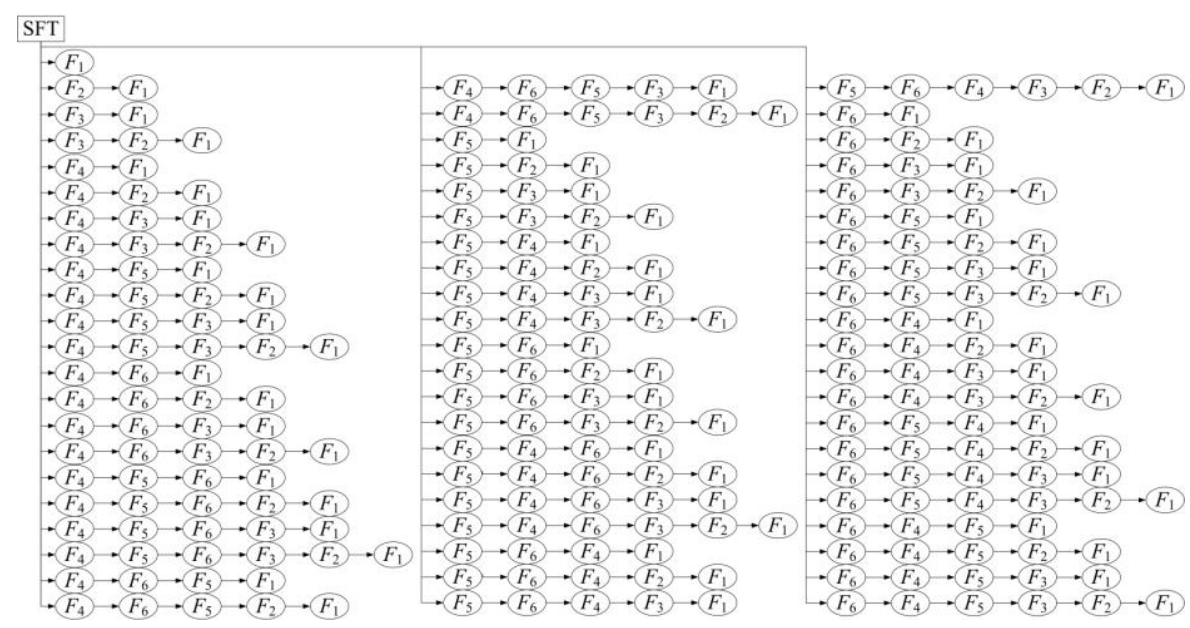

Fig. 14. The SFT of Design-I. 
In the SFT of Design-I, there are 64 branches and 260 nodes, and the average length of branches is 4.0625 , i.e., $b_{k}=64, N_{k}=260, L_{\text {avg }}=4.0625$. The shortest branch is ' $F_{1}$ ', so the antishake module is the weakness of a continuous diagnosis.

\subsubsection{Direct calculation method}

Using the direct calculation method, the metrics can be calculated based on the DGM and the D matrix without establishing an SFT.

As shown in Fig. 13, the D matrix of Design-I is an upper triangular matrix. The values of D matrix metric were calculated according to (14) in section 4.3 and the results are shown in Table 1 . Because the value of $\left\|F_{1}\right\|_{1}$ is the maximum in the 1-norms, the anti-shake module corresponding to $F_{1}$ is the weakness of a continuous diagnosis

According to (18), (22) and (23), we have achieved the values of SFT metrics, as shown in Table 1.

Compared with the SFT searching method, the direct calculation method can achieve the same results without establishing and searching an SFT.

Table 1. Metric values of Design-I.

\begin{tabular}{|c|c|c|c|c|c|c|c|c|}
\hline \multicolumn{6}{|c|}{ D matrix metric } & \multicolumn{3}{|c|}{ SFT metrics } \\
\hline$\left\|F_{1}\right\|_{1}$ & $\left\|F_{2}\right\|_{1}$ & $\left\|F_{3}\right\|_{1}$ & $\left\|F_{4}\right\|_{1}$ & $\left\|F_{5}\right\|_{1}$ & $\left\|F_{6}\right\|_{1}$ & $b_{k}$ & $N_{k}$ & $L_{\mathrm{avg}}$ \\
\hline 6 & 5 & 4 & 1 & 1 & 1 & 64 & 260 & 4.0625 \\
\hline
\end{tabular}

\subsection{Design-II}

The DGM and corresponding D-matrix are shown in Figs. 15 and 16. The DGM of Design-II is also a typical fore-series-aft-parallel DGM in which four faults are connected in series with two faults connected in parallel, i.e., $k 1=4, k 2=2$.

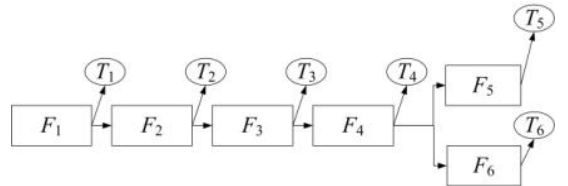

Fig. 15. The DGM of Design-II.

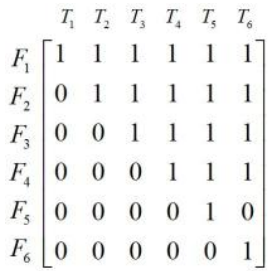

Fig. 16. The D matrix of Design-II.

\subsubsection{SFT searching method}

Using the SFT searching method, we have established the SFT of design-II, which is shown in Fig. 17.

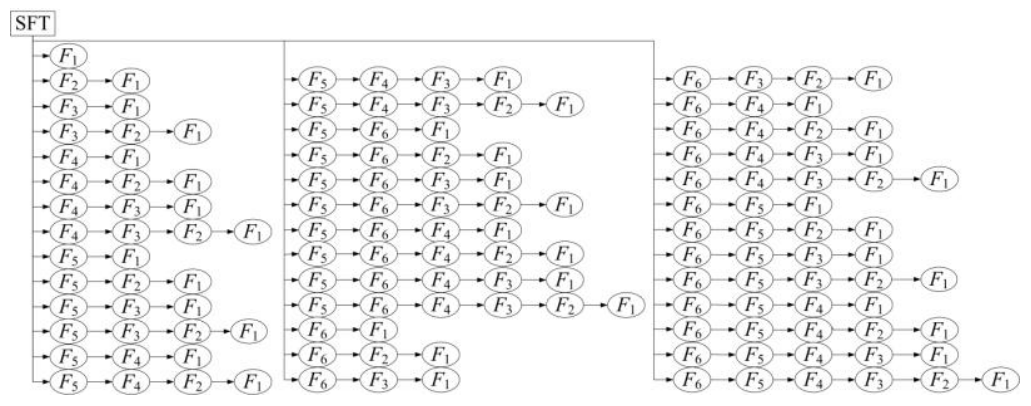

Fig. 17. The SFT of Design-II. 
In the SFT of Design-II, there are 40 branches and 148 nodes, and the average length of branches is 3.7, i.e., $b_{k}=40, N_{k}=148, L_{\mathrm{avg}}=3.7$. The shortest branch is ' $F_{1}$ ', so the anti-shake module is also the weakness of a continuous diagnosis.

\subsubsection{Direct calculation method}

As shown in Fig. 16, the D matrix of Design-II is an upper triangular matrix. The values of D matrix metric were calculated according to (14) in section 4.3 and the results are shown in Table 2. Because the value of $\left\|F_{1}\right\|_{1}$ is the maximum in the 1-norms, the anti-shake module corresponding to $F_{1}$ is the weakness of a continuous diagnosis

According to (18), (22) and (23), we have achieved the values of SFT metrics, as shown in Table 2.

Table 2. Metric values of Design-II.

\begin{tabular}{|c|c|c|c|c|c|c|c|c|}
\hline \multicolumn{7}{|c|}{ D matrix metric } & \multicolumn{3}{c|}{ SFT metrics } \\
\hline$\left\|F_{1}\right\|_{1}$ & $\left\|F_{2}\right\|_{1}$ & $\left\|F_{3}\right\|_{1}$ & $\left\|F_{4}\right\|_{1}$ & $\left\|F_{5}\right\|_{1}$ & $\left\|F_{6}\right\|_{1}$ & $b_{k}$ & $N_{k}$ & $L_{\text {avg }}$ \\
\hline 6 & 5 & 4 & 3 & 1 & 1 & 40 & 148 & 3.7 \\
\hline
\end{tabular}

\subsection{Results analysis}

Using $L_{\text {avg, }}$, the continuous diagnosis capabilities of both designs can be measured and compared to determine a priority selection.

In Design-I, $L_{\mathrm{avg}}=4.0625$; and in Design-II, $L_{\mathrm{avg}}=3.7$. So the average length of branches of Design-I is greater than that of Design-II, and Design-I is the priority selection.

Additionally, the weakness of a continuous diagnosis can be determined by using the D matrix metric. In both designs, the values of $\left\|F_{1}\right\|_{1}$ are the maximum, thus the anti-shake module corresponding to $F_{1}$ is the weakness, and it should be designed of high reliability.

\section{Conclusions}

Sequential fault combination is a typical form of multi-faults. A dependency matrix can be applied to perform the continuous diagnosis of a system. How to measure the continuous diagnosis capability is worth considerable research effort. All of the sequential fault combinations of a system can be described as a sequential fault tree. A continuous diagnosis capability can be measured by establishing and searching a sequential fault tree to select an optimized design, and the best length metric is $L_{\text {avg. }}$. For a series DGM, a parallel DGM and a fore-series-aft-parallel DGM, the metric calculation models of $b_{k}, N_{k}$ and $L_{\text {avg }}$ are derived to measure continuous diagnosis capabilities directly, which can achieve the same results with high efficiency. Further effort should be devoted to establish more direct calculation models to measure the continuous diagnosis capabilities for complex DGM types. In addition, failure probabilities have an important influence on a continuous diagnosis capability and should be introduced in the direct calculation method to accomplish a more accurate decision.

\section{References}

[1] Emment, G. (2010). Method for improving design testability through modelling. In Proc. IEEE AUTOTESTCON, 1-4.

[2] Ma, F., Song, D. (2010). A new testability model of electronic system and its application. In Proc. 2010 International Conference on Computer Application and System Modelling (ICCASM), 10, 516-520. 
[3] Shi, J.Y., Gong, J.J. (2010). Improvement method for testability modelling with multiple faults. Journal of Beijing University of Aeronautics and Astronautics, 36(30), 270-273.(in Chinese)

[4] Temple, G., Jize, N. (2005). Testability modelling and analysis of a rocket engine test stand. In Proc. IEEE Aerospace Conference, 3874-3895.

[5] Rao, N.S.V. (1996). On parallel algorithms for single-fault diagnosis in fault propagation graph systems. IEEE Trans. Parallel and Distributed Systems, 7(12), 1217-1223.

[6] Filippetti, F., Artioli, M. (2000). Single and multiple fault diagnosis based on symbolic analysis and reduced set of observable points for linear analogue circuits. In Proc. 7th IEEE International Conference on Electronics, Circuits and Systems (ICECS), 433-436.

[7] Anita, J.P., Vaathi, P.T. (2010). Multiple fault diagnosis with improved diagnosis resolution for VLSI circuits. In Proc. 2010 International Conference on Computer Communication and Networking Technologies (ICCCNT), 1-6.

[8] Kundu, S., Chattopadhyay, S. (2011). Multiple fault diagnosis based on multiple fault simulation using particle swarm optimization. In Proc. 24th International Conference on VLSI Design, 364-369.

[9] Kleer, J.D., Williams, B.C. (1987). Diagnosing multiple faults. Artif. In-tell.

[10] Simpson, W.R., Sheppard, J.W. (1994). Multiple failure diagnosis. In Proc. of the IEEE AUTOTESTCON, 381-389.

[11] Shakeri, M., Pattipati, K.R. (1998). Optimal and near-optimal algorithms for multiple fault diagnosis with unreliable tests. IEEE Trans. SMC, 28(3), 431-443.

[12] Fang, T., Pattipati, K.R. (2003). Computationally efficient algorithms for multiple fault diagnosis in large graph-based systems. IEEE Trans. SMC, 33(1), 73-82.

[13] Tung, L., Hadjicostis, C.N. (2008). Max-product algorithms for the generalized multiple-fault diagnosis problem. IEEE Trans. Systems, Man, and Cybernetics, Part B, Cybernetics, 3(3), 1607-1621.

[14] Tadeusiewicz, M., Hałgas, S. (2005). Multiple fault diagnosis in analogue circuits. In Proc. of the 2005 European Conference on Circuit Theory and Design, 205-208.

[15] Wang, Z., Marek-Sadowska, M. (2003). Multiple fault diagnosis using n-detection tests. In Proc. of 21st International Conference on Computer Design, 198-201.

[16] Anita, J.P., Vaathi, P.T. (2010). Multiple fault diagnosis with improved diagnosis resolution for VLSI circuits. In Proc. 2010 International Conference on Computing Communication and Networking Technologies (ICCCNT), 1-6.

[17] Grzechca, D., Rutkowski, J. (2009). Fault diagnosis in analogue electronic circuits- the SVM approach. Metrol. Meas. Syst., 16(4), 583-597.

[18] Lu, Y., Gan, Z.H. (2010). Research and implementation of a multi-fault diagnosis method based on casebased reasoning. In Proc. 8th World Congress on Intelligent Control and Automation (WCICA), 5737-5741.

[19] Luo, Z.G., Chen, Q. (2010). Multi-fault diagnosis of gear based on sequential fuzzy inference. In Proc. 2010 International Conference on Mechanic Automation and Control Engineering (MACE), 2492-2496.

[20] Grzechca, D. (2011). Soft fault clustering in analogue electronic circuits with the use of self organizing neural network. Metrol. Meas. Syst., 18(4), 555-568.

[21] Lin, Y.C., Lu, F. (2007). Multiple-fault diagnosis based on adaptive diagnostic test pattern generation. IEEE Trans. Computer-Aided Design of Integrated Circuits and Systems, 26(5), 932-942.

[22] Tadeusiewicz, M., Hałgas, S. (2011). Multiple soft fault diagnosis of nonlinear DC circuits considering component tolerances. Metrol. Meas. Syst., 18(3), 349-360.

[23] Shi, J.Y., Lin, X.G., Lv, K.Y. (2012). A method for searching and evaluating diagnosable sequence fault sets of a dependency matrix. In Proc. IEEE Conference on Prognostics \& System Health Management, 1-7.

[24] Singh, S., Holland, S.W., Bandyopadhyay, P. (2010). Trends in the development of system-level fault dependency matrices. In Proc. IEEE Aerospace Conference, 1-9. 